\title{
Closed-loop Simulation of Buck Converter Based on PSIM
}

\author{
Sun Quan ${ }^{1, a^{*}}$, Sun Yuan ${ }^{2, b}$ \\ ${ }^{1}$ School of Mechanical Engineering, Shanghai Institute of Electrical Engineering, Shanghai, China \\ ${ }^{2}$ School of Mechanical Engineering, Shanghai Institute of Electrical Engineering, Shanghai, China
}

\begin{abstract}
This paper introduces the working principle of Buck converter under voltage control, and studies the modeling method of Buck converter under special power electronics simulation software. Finally, the simulation of the converter is carried out under the condition of the mutation of input voltage and load. The simulation results show that the voltage control strategy has good transient response and anti-jamming ability, which has a good guiding significance for practical application.
\end{abstract}

\section{The introduction}

First of all, with the rapid development of science and technology, switching power supply technology is applied in more and more scenarios such as aviation secondary power supply, all kinds of fast charging and other fields. One of the ways to improve the power density of the switching power supply is high-frequency, that is, to improve the working efficiency of the PWM converter, so as to reduce the volume weight of the energy storage components in the circuit ${ }^{[1-7]}$. The single control feedback strategy in switching power supply cannot meet the requirement of power electronics design because of the slow transient response speed ${ }^{[2]}$. According to the difference of current sampling signal, the current control system can be divided into peak current control, average current control and valley current control. Advantages of peak current control: (1) Simple current limiting protection; (2) Soft starting circuit can not be added in the peak current control, but must be added in the voltage type control. Here we choose voltage - type control to do specific analysis.

\section{Buck converter in voltage control mode}

\subsection{Main circuit parameters and working principle}

The control object discussed in this paper is Buck converter, which must realize the DC conversion function with switching frequency of $100 \mathrm{kHz}$, input of $12 \mathrm{~V}$ and output of $5 \mathrm{~V} / 50 \mathrm{~W}$. Figure 1 shows the main circuit model of Buck converter. When designing BUCK converter, it is required to operate in inductive current continuous mode, and the output voltage ripple is not more than $0.5 \%$ of the output voltage. After calculation, when the main circuit inductance, capacitance can meet the requirements.
BUCK converter is a non-isolated converter, which can obtain the low voltage required by the load by passing the input high voltage through the switching and cutoff of the power switch. Its structural topology is shown in Figure 1.

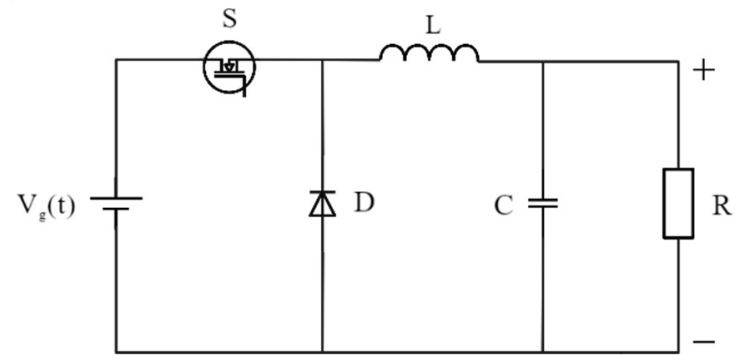

Fig. 1 Peak current control step-down circuit schematic diagram

According to the topology of the converter, its basic working principle is as follows: when the switch $\mathrm{S}$ is turned on, the diode $\mathrm{D}$ is subjected to the back voltage cutoff, and the circuit's working path is to charge the capacitor $\mathrm{C}$ and supply the load. $\mathrm{V}_{\mathrm{g}}(\mathrm{t})-\mathrm{S}-\mathrm{L}$ The current across the inductor increases linearly, so the inductor stores energy. In one switching cycle, the switching tube $\mathrm{S}$ conduction time is. $T_{\mathrm{s}}$ When the switch $\mathrm{S}$ is turned off, the current on the inductor cannot change abruptly, so that the current continues through the diode. The circuit's working path is D-L to supply power to the load. The inductor current drops linearly, and the inductor is discharged. Within a switching cycle, the switching tube $\mathrm{S}$ shutoff time is. $\mathrm{T}_{\mathrm{s}} \mathrm{T}_{\text {off }}$ Thus, a modal diagram of the Buck converter can be drawn, as shown in Figure 2. 


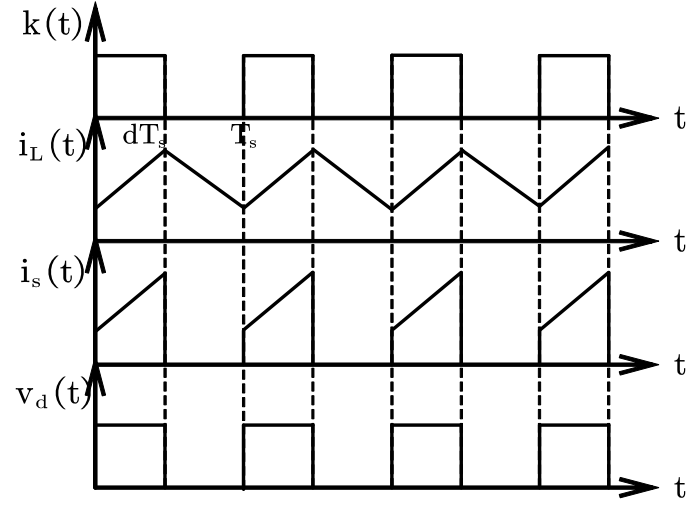

Fig. 2 Modal diagram of step-down circuit

\subsection{Modeling of voltage closed loop small signal}

The establishment of the real power level transfer function of the main circuit is generally stateless space averaging method, discrete averaging method, sampling data model, equivalent power averaging method, three-terminal PWM switch model, etc.The equivalent average power method is a circuit analysis method proposed to simplify the operation of the state space average method and popularize it. The basic idea of the method is that the actual switches of PWM DC-DC power converters are respectively equivalent with corresponding controlled sources to obtain the equivalent controlled source circuit model of the power converters. Then all the variables in the equivalent controlled source circuit model and the controlled source are averaged in the switching cycle to obtain the average controlled source circuit model of the actual circuit. Then the average of the controlled source circuit model circuit variables as steady state and small signal disturbances of the special dynamic variables, and the average controlled source circuit model of the nonlinear controlled source variable linearization, after linearization of the equivalent controlled source circuit using the superposition principle, break it down into a steady state and dynamic small signal two sub circuit;Finally, the steady-state working point relation of the power converter is obtained by the steady-state equivalent subcircuit, and the small signal transfer function of the power converter at the steady-state working point is obtained by the operation circuit of the dynamic small signal subcircuit. The following models and conclusions are based on the equivalent average power method.

The steady-state operating point converter model is shown in Fig. 3.

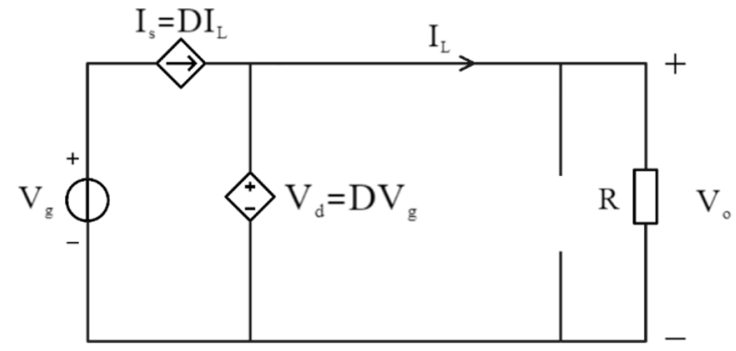

Fig. 3 Steady-state operating point model

Small signal model of steady-state operating point is shown in Fig. 4:

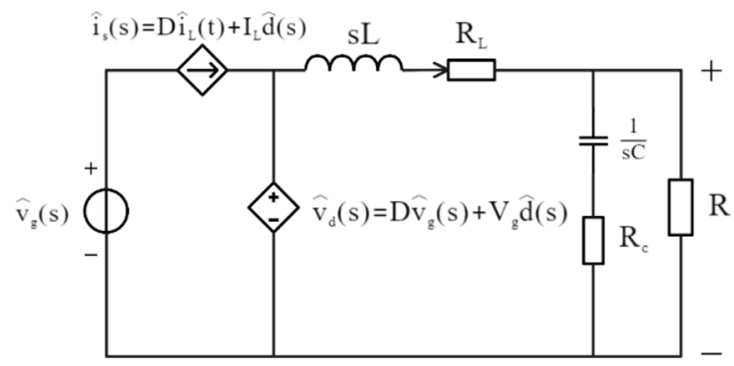

Fig. 4 Small signal model of steady-state operating point

Let the small signal disturbance of input voltage be zero, and list the $\mathrm{KCl}$ and $\mathrm{KVL}$ equations of the circuit, so that:

$$
\mathrm{G}_{\mathrm{vd}}(\mathrm{s}) \triangleq \frac{\hat{\mathrm{v}}_{\mathrm{o}}}{\hat{\mathrm{d}}}=\mathrm{V}_{\mathrm{g}} \frac{\mathrm{sR}_{\mathrm{c}} \mathrm{C}+1}{\mathrm{~s}^{2} \mathrm{LC}+\mathrm{s}\left[\left(\mathrm{R}_{\mathrm{L}}+\mathrm{R}_{\mathrm{c}}\right) \mathrm{C}+\mathrm{L} / \mathrm{R}\right]+1}
$$

Different control strategies are constructed according to different signals connected to the reverse end of the modulator. For example: the reverse end of the modulator is connected to the fixed slope voltage signal is the voltage type control; Peak current control can be constituted by inverting inductive current signal of the modulator. A current amplifier is indirectly connected between the inphase end of the modulator and the output end of the error amplifier. The inverting end of the current amplifier is the inductive current signal. At the same time, the inverting end of the modulator is connected with the fixed slope voltage signal, which constitutes the average current control. The inverter terminal of the modulator is connected to the output voltage, and the output voltage ripple is used as the equivalent oblique wave, which constitutes the control and so on. There are many other control methods, and we will focus on voltage control next. $\mathrm{V}^{2}$

According to paper [1], the small-signal equation of voltage-controlled modulator is as follows:

$$
\hat{\mathrm{d}}=\mathrm{F}_{\mathrm{m}} \hat{\mathrm{v}}_{\mathrm{c}}
$$

Where, is the peak value of the fixed slope voltage signal connected to the inverting terminal of the comparator. $\mathrm{F}_{\mathrm{m}}=\frac{1}{\mathrm{~V}_{\mathrm{m}}} \mathrm{V}_{\mathrm{m}}$

Substitute Equation (2) into Equation (1), and we can get:

$$
\mathrm{G}_{\mathrm{vc}}^{\prime}(\mathrm{s}) \triangleq \frac{\hat{\mathrm{v}}_{\mathrm{o}}}{\hat{\mathrm{v}}_{\mathrm{c}}}=\mathrm{FmV}_{\mathrm{g}} \frac{\mathrm{sR}_{\mathrm{c}} \mathrm{C}+1}{\mathrm{~s}^{2} \mathrm{LC}+\mathrm{s}\left[\left(\mathrm{R}_{\mathrm{L}}+\mathrm{R}_{\mathrm{c}}\right) \mathrm{C}+\mathrm{L} / \mathrm{R}\right]+1}(3)
$$




\section{Simulation of 2.3 .}

Draw Bode diagram in MATLAB, as shown in Figure 5.

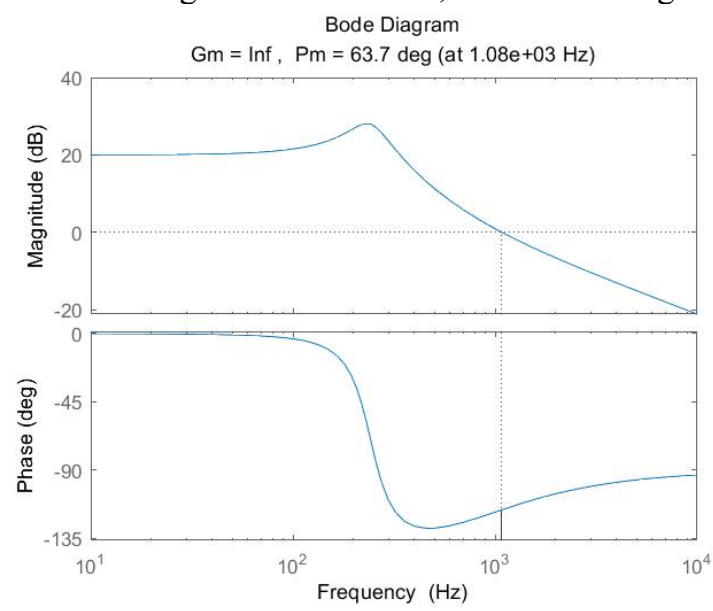

Fig. 5 Modal diagram of step-down circuit

As can be seen from Fig. 5, the crossing frequency is $1.08 \mathrm{kHz}$ and the phase margin. $\mathrm{P}_{\mathrm{m}}=63.7^{\circ}$ Although the stability margin of the system meets the requirements of stability, if the parameters in the system change slightly. The system may become unstable; DC gain, then the steady-state error of the system is, which is acceptable in engineering. $\mathrm{T}_{\mathrm{uo}}=201 /\left(1+\mathrm{T}_{\mathrm{u} 0}\right)=4.8 \%$ In addition, the crossing frequency is $1.08 \mathrm{kHz}$, and the response speed of the system is slow.It can not meet the requirements of stable, accurate and fast control object, so it is necessary to design a reasonable compensation network to make the Buck converter work stably.

Single closed-loop voltage control is shown in Fig. 6.

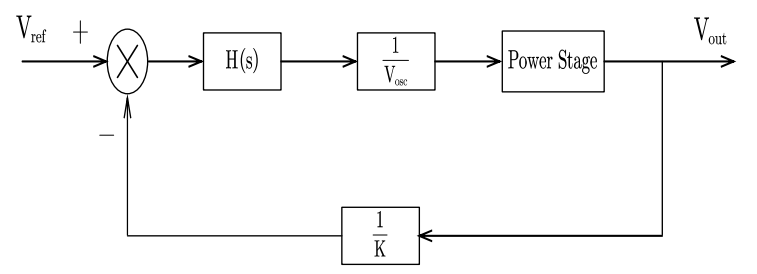

Fig. 6 Voltage single closed loop control

OPA III compensator was selected, as shown in Figure 7.

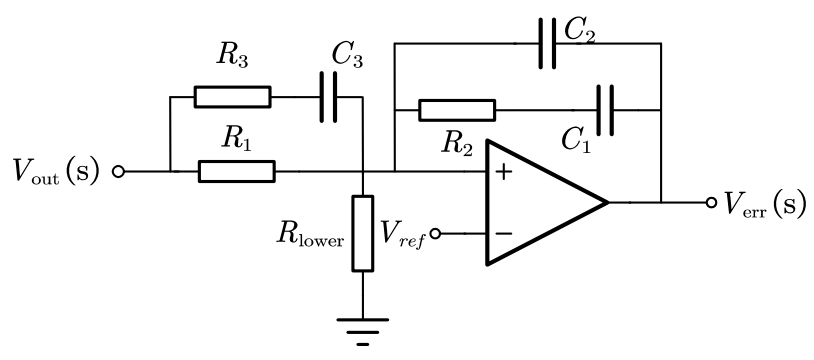

Fig. 7 Type III compensator of OPA

After adding the compensator, the simulation schematic diagram we built is shown in Figure 8.

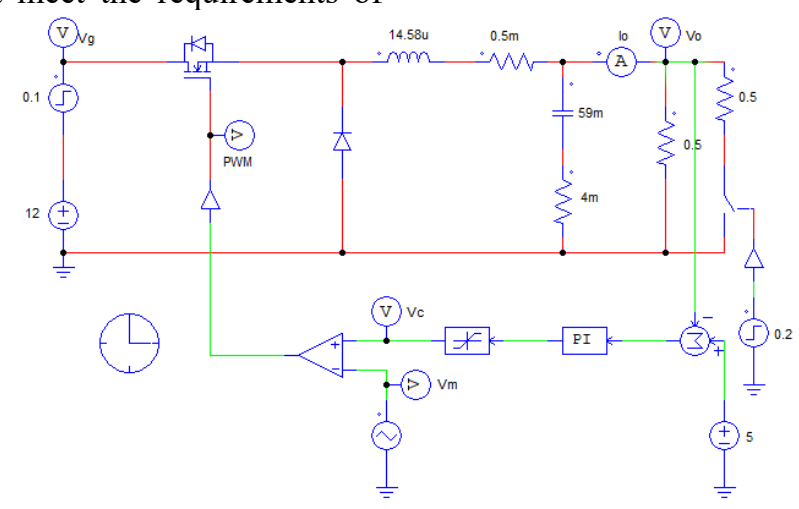

Fig. 8 Voltage closed-loop simulation schematic diagram

The simulation structure is shown in Figure 8.

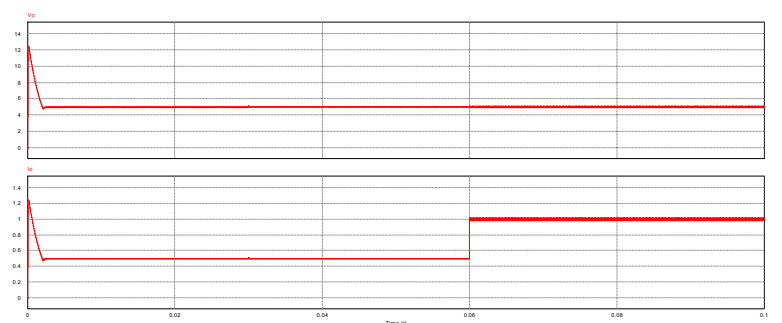

Fig. 9 Voltage closed-loop simulation schematic diagram

\section{Conclusion}

The simulation results show that the voltage control has good transient response and stability.Because the current signal does not participate in the feedback, the system will not be disturbed by the current noise. Duty cycle adjustment is not restricted; Feedback design is easy to adjust; Quick response to output load changes; Low output impedance;At the same time, it also has some 
disadvantages, in the single feedback loop control system, the output LC filter produces double poles in the control loop, the dynamic response is slow, and a zero point is needed to compensate the main pole, the feedback compensation design is relatively complex, more additional devices are needed to design the compensation loop, in order to optimize the transient response of the load.In general, its control strategy also applies to other topologies.

\section{reference}

1. Feng Quanyuan, Yan Hongju.Design of a Variable Parameter Proportional Integral Control BUCK Converter $[\mathrm{J}]$. Electric Machines and Control, 2016,20 (5):68-76.

2. Wang Lianghua, Yue Jiguang, Su Yongqing, et al. Design of Average Current Control BUCK Converter Control System $[\mathrm{J}]$. Chinese Journal of Power Sources, 2013, 37 (12):2225-2228.

3. Xu Lin, Dai Wei, Geng Pan, et al. Influence of Double Closed-loop Control on Dynamic Response of Buck Converter [J]. Ship Electric Technology, 201, 41(1) : 11-15.

4. Gao Benyou, Zhang Weiping, Zhang Xiaoqiang.Simulation Research of Double Closedloop Control System Design Based on BUCK Circuit [J]. Electronic World, 2017, 03; 125-127.

5. Chen Yao-zhi. Method Analysis of Small Signal Modeling for Switching Power Supply [J]. Neijiang Science and Technology, 2015,36 (11) : 57-58.

6. Zang Yue, Wang Jian. Modeling and Analysis of V2C Controlled Step-down Converter $[\mathrm{J}]$. Industrial Control Computer, 2017,3,15:142-143.

7. Suping Huang. Research and design of Buck converter loop stability. Master's Dissertation of Southwest Jiaotong University.2014. 